\title{
Breakdown of Hund's third rule for intrinsic magnetic moments
}

\author{
Julia Herrero-Albillos \\ Helmholtz-Zentrum Berlin für Materialien und Energie GmbH, \\ Albert-Einstein-Str. 15, 12489 Berlin, Germany \\ L. M. García and F. Bartolomé \\ Instituto de Ciencia de Materiales de Aragón, and Dpto. Física de la Materia Condensada, \\ CSIC-Universidad de Zaragoza 50009, Zaragoza, Spain
}

\author{
A. T. Young \\ Advanced Light Source, Lawrence Berkeley National Laboratory, \\ University of California, Berkeley, California, 94720, USA
}

(Dated: October 4, 2012)

\begin{abstract}
Detailed X-ray magnetic circular dichroism measurements as a function of temperature are reported in $\mathrm{ErCo}_{2}$ at the Co $\mathrm{L}_{2,3}$ edges. The application of magneto-optical sum rules allows for the determination of the Co orbital and spin magnetic moments both in the ferrimagnetic and paramagnetic phases. In agreement with the predictions of Hund's third rule, Co orbital and spin moments are parallel through out the overall temperature range, except in a narrow temperature window within the paramagnetic phase in which, contrary to what is expected, they are overall coupled antiparallel. This is the first time such behavior is observed in an intrinsic magnetic moment. A qualitative consideration of the balance between the inter-atomic and intra-atomic interactions acting on the cobalt atoms explains the phenomenon.
\end{abstract}

PACS numbers:

Hund's rules are empirical laws developed in the framework of atomic physics to determine the ground state of a multi-electron atom assuming a Russell-Saunders coupling [? ? ]. The standard textbook interpretation based on quantum mechanics is due to Slater [? ]. Hund's rules were originally developed in an atomistic picture, and it is therefore surprising that they also hold almost universally for molecules and solids. However, in 1993 A. Hjelm et al. [? ] predicted, by means of spin polarized electronic structure calculation, a breakdown of Hund's third rule for the induced magnetism in uranium metal. According to these calculations, uranium spin and orbital moments should be coupled parallel rather than antiparallel as stipulated by Hund's third rule. Furthermore, they conjectured that the field induced spin and orbital moments of paramagnetic metals should be parallel, irrespective of the filling of the electronic shell, except in the case of systems with short-range order [? ]. A decade later, in a work based on first principle calculations, Galanakis et al.[? ? ] proposed the intermetallic compounds $\mathrm{VAu}_{4}$ and $\mathrm{VPt}_{3}$ as good candidates to violate the Hund's third rule due to the influence of ligand states and later on they suggested the same phenomenon for the $3 \mathrm{~d}$ Cr magnetic moments in $\mathrm{CrPt}_{3}$ compounds.[? ] Another systematic theoretical study by Tyer et al.[? ] of the layer resolved spin and orbital magnetic moments of $4 \mathrm{~d}$ and $5 \mathrm{~d}$ at the interface with Fe films also reported an expected violation of Hund's third rule due to the hybridization between the $5 \mathrm{~d} / 4 \mathrm{~d}$ and the Fe.

In general, once the atoms are immersed in a solid, the delocalization of electron wave functions could result in inter-atomic spin-other-orbit coupling overcoming the normal one-electron spin-orbit coupling, therefore changing the ground state relative alignement between spin and orbital moments. However, there have been very few experiments showing a breakdown of the Hund's third rule. Only in recent decades, highly sensitive and element specific magnetometries like x-ray magnetic circular dichroism (XMCD) have allowed the separated detection of small induced magnetic moments in nanostructured magnetic systems [? ? ? ]. Additionally, the application of the magnetooptical sum rules [? ? ] to the XMCD spectra allows the separate determination of the orbital and the spin magnetic moments. Using these tools, Wilhelm et al. [? ] observed experimentally in 2001 the violation of Hund's third rule in $\mathrm{W}$ induced-moments in a $\mathrm{W} / \mathrm{Fe}$ multilayer system (with nominal $0.5 \mathrm{~nm} \mathrm{~W}$-thickness). This result was in agreement with previous first-principles theoretical calculations [? ? ? ]. In a previous work, Schütz and coworkers [? ] obtained XMCD experimental and theoretical values for magnetic moments of $5 d$ impurities in Fe, showing some discrepancies with the Hund's third rule predictions (for Os, Re and Ir). In 2006, V.V. Krishnamurthy et al.,[? ] confirmed, also by XMCD, the violation of Hund's third rule of Ir 5d induced magnetic moments in Co-Ir alloys.

The work by Wilhelm et al. [? ] opened an interesting debate [? ? ] between theoreticians and experimentalists about the relevance of local structure in these phenomena. In particular, it was clearly established that the case of induced magnetism in alloys is different from that on layered structures (see Ref. [? ]). However, the debate has been always focused in $5 d$ induced magnetic moments. In the case of $3 d$ moments, the relative orientation between spin and 
FIG. 1: (Color online) $\mathrm{ErCo}_{2} \mathrm{XMCD}$ spectra (left) and the integrals of the fitted data (right) at the Co $\mathrm{L}_{2,3}$ edges at selected temperatures near $T_{\mathrm{F}}$ at $1 \mathrm{~T}$. Arrows indicate the alignment of $m_{\mathrm{L}}$ and $m_{\mathrm{S}}$ as obtained from the sum rules.

FIG. 2: (Color online) Co $3 d$ band spin and orbital moments at $1 \mathrm{~T}$ from $20 \mathrm{~K}$ to $100 \mathrm{~K}$ (main graph) and in the proximities of $T_{\mathrm{F}}$ (inset) obtained from the application of the sum rules to the $\mathrm{Co}_{2,3} \mathrm{XMCD}$ edges of ErCo $\mathrm{Co}_{2}$ Note that in the inset $m_{\mathrm{L}}$ is plotted against the left axes (blue) and $m_{\mathrm{S}}$ against the right axes (red).

orbital magnetic moments were always reported to obey Hund's third rule [? ? ]. In this letter we demonstrate, by means of XMCD measurements, the existence of a breakdown of Hund's third rule in an intrinsic magnetic moment, namely in the $3 d$ moment of a Co Laves phase compound. In particular, we have detected this phenomenon in a narrow temperature range within the paramagnetic phase of $\mathrm{ErCo}_{2}$ in which very strong short-range correlations have been identified [? ]. To our knowledge, this is the first evidence of a violation of Hund's third rule in an intrinsic magnetic moment of a solid.

The $\mathrm{ErCo}_{2}$ polycrystalline ingots used in this work have been thoroughly characterized in previous works. Experimental details of sample preparation together with a complete structural and magnetic characterization can be found

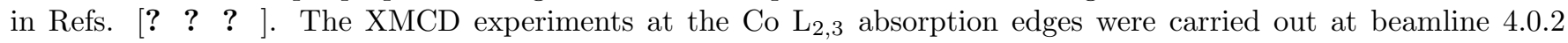
at Advanced Light Source using a low-temperature end station equipped with a superconducting magnet [? ? ? ]. Details of the data acquisition and data treatment can be found elsewhere [? ? ].

The magnetism in $\mathrm{ErCO}_{2}$ is dominated by the Er sublattice, with an essentially temperature independent magnetic moment of around $8.8 \mu_{B}$ /atom. The magnetic order is set through a structural first-order transition $\left(T_{\mathrm{c}}=35 \mathrm{~K}\right.$ at $H=1 \mathrm{~T}$ ). It is well known that below this temperature, the Co magnetic sublattice is ordered antiparallel to the Er one with an induced magnetic moment of around $0.8 \mu_{B}$ /atom, giving rise to a ferrimagnetic system. The nature of Co magnetic moment in the paramagnetic phase has been a matter of debate for decades. Combining XMCD, Small Angle Neutron Scattering and a.c. magnetic susceptibility measurements we were able to prove the existence of a 0.2 $\mu_{B} /$ atom Co intrinsic magnetic moment [? ? ]. That intrinsic Co moment changes its orientation relative to the applied magnetic field and the Er moments at a temperature $\left(T_{\mathrm{F}}=60 \mathrm{~K}\right.$ at $\left.H=1 \mathrm{~T}\right)$ much higher than the ordering temperature. The region delimited by $T_{\mathrm{c}}$ and $T_{\mathrm{F}}$ corresponds to a new magnetic arrangement -denoted parimagnetic phase- in which Co moments are disordered (as in the standard paramagnetic phase) but oriented antiparallel in average to the Er moments (as in the ferrimagnetic phase). More recently, we have been able to correlate $T_{\mathrm{F}}$ with the formation of a Griffiths-like phase [?] in $\mathrm{ErCo}_{2}$. Indeed, the change of sign of the Co magnetization at $T_{\mathrm{F}}$ is driven by the formation of Co magnetic clusters which are ferromagnetically coupled within the paramagnetic phase [? ? ].

Figure ?? shows selected XMCD spectra and their integrals obtained at the $\mathrm{Co}_{2,3}$ edges from temperatures just above $T_{\mathrm{c}}$ to well above $T_{\mathrm{F}}$ at $1 \mathrm{~T}$. For those XMCD spectra where the signal to noise ratio is low (i.e. near $T_{\mathrm{F}}$ ), the integrals have been evaluated from the fit of the XMCD spectra to two independent peaks (one for each edge) with the same energy dependence as those at low temperatures, where the noise is less that $1 \%$ of the maximum signal. By applying the sum rules [? ? ] to the XMCD spectra the Co orbital and spin moments projected along the applied magnetic field direction $\left(m_{\mathrm{L}}\right.$ and $m_{\mathrm{S}}$ respectively) are obtained separately. The standard approximations for $3 d$ metals and cubic systems [? ? ? ? ? ] have been assumed in this work [? ? ]. Therefore, the contribution of the dipolar operator $\mathrm{T}_{z}$ to the effective spin given by the spin sum rule has been neglected. The temperature evolution of the orbital and spin Co moments obtained for $\mathrm{ErCo}_{2}$ at $1 \mathrm{~T}$ are shown in figure ??. The overall temperature behaviour is very similar to what has been already reported for the total Co $3 d$ magnetic moment in this compound [? ? ]: above $T_{\mathrm{F}}$ both $m_{\mathrm{L}}$ and $m_{\mathrm{S}}$ are parallel to the applied magnetic field while in the parimagnetic and ferrimagnetic phases they are antiparallel to the applied magnetic field. Abrupt jumps in magnitude can be observed at $T_{\mathrm{c}}$ and $T_{\mathrm{F}}$ for both moments. The overall parallel alignment between $m_{\mathrm{L}}$ and $m_{\mathrm{S}}$ is in acordance with Hund's third rule prediction.

In what follows we will focus on the relationship between $m_{\mathrm{L}}$ and $m_{\mathrm{S}}$. It is convenient then to consider the different weights the integrals of XMCD at the $\mathrm{L}_{3}$ and $\mathrm{L}_{2}$ edges ( $A$ and $B$ respectively, see figure ??) have in the determination of $m_{\mathrm{L}}$ and $m_{\mathrm{S}}$. It follows from the sum rules that $m_{\mathrm{L}} \propto-2(A+B) / 3$ and $m_{\mathrm{S}} \propto 2 B-A$ [? ]. Note that a spectrum with $A=-B$ implies a spin-only moment (as found in $\mathrm{ErCo}_{2}$ at $T=59.3 \mathrm{~K}$ ), while $A=2 B$ implies an orbital-only moment (as nearly found at $T=61.8 \mathrm{~K}$ ).

While the observed spectra at temperatures below $45 \mathrm{~K}$ and above $68 \mathrm{~K}$ are typical for metallic systems [? ], that is not the case for the spectra collected in the vicinity of $T_{\mathrm{F}}$, well within the paramagnetic phase. Indeed, a 


\begin{tabular}{|l|c|c|cc|c|c|c|}
\hline & \multicolumn{2}{|c|}{ Orbital } & & \multicolumn{3}{|c|}{ Spin } \\
\cline { 2 - 7 } & $\mathrm{H}_{a p p}$ & $\mathrm{H}_{L S}$ & $m_{L}$ & $m_{S}$ & $\mathrm{H}_{a p p}$ & $\mathrm{H}_{L S}$ & $\mathrm{H}_{E x}^{E r}$ \\
\hline Low T & $\uparrow$ & $\Downarrow$ & $\downarrow$ & $\downarrow$ & $\uparrow$ & $\downarrow$ & $\Downarrow$ \\
\hline $\mathrm{T} \simeq \mathrm{Tf}$ & $\Uparrow$ & $\downarrow$ & $\uparrow$ & $\downarrow$ & $\uparrow$ & $\uparrow$ & $\Downarrow$ \\
\hline High T & $\Uparrow$ & $\uparrow$ & $\uparrow$ & $\uparrow$ & $\Uparrow$ & $\uparrow$ & $\downarrow$ \\
\hline
\end{tabular}

TABLE I: Diagram illustrating the balance of fields acting on the Co orbital and spin moments below, near and above $T_{\mathrm{F}}$, where their orientations are indicated by the arrows and double arrows are used for the governing interaction.

FIG. 3: (Color online) Third components of the orbital vs. spin magnetic moments in the Co $3 d$ band of ErCo 2 at 1 T. A fit of the experimental data to a straight line is shown.

close look into the XMCD spectra and their integrals in figure ?? reveals fascinating phenomena. First, spectrum at $62.5 \mathrm{~K}$ correspond to a moment with a huge orbital contribution, quite similar to what has been reported in Co single atoms deposited on $\mathrm{Pt}$, where strongly unquenched orbital Co moments are present [? ]. Second, the XMCD spectrum at $59.3 \mathrm{~K}$ is a clear example of a small spin-only magnetic moment antiparallel to the applied field with no orbital moment $(A=-B)$. This case is more common in magnetism, as orbital moments are usually quenched in metallic systems. Last but not least -and more surprisingly- spectra at $60.6 \mathrm{~K}$ and $61.1 \mathrm{~K}$ correspond to a Co magnetic moment where the orbital component is of opposite sign to the spin component. These spectra are two selected examples providing a breakdown of the Hund's third rule (note that this can be already observed by mere visual inspection of the spectra). The inset of figure ?? shows the numerical values of $m_{\mathrm{L}}$ and $m_{\mathrm{S}}$ obtained from $\mathrm{XMCD}$ in the vicinity of $T_{\mathrm{F}}$, where the breakdown takes place. In fact, the physical phenomena leading to the above described spectra lies on the different temperatures at which the Co spin and orbital moments change their sign: an antiparallel alignment of $m_{\mathrm{L}}$ and $m_{\mathrm{S}}$ can be observed in a temperature window of around $3 \mathrm{~K}$.

In order to understand the phenomena we must take into account the fields acting on $m_{\mathrm{L}}$ and $m_{\mathrm{S}}$. Two fields determine the Co orbital moment orientation: the external field, $\mathrm{H}_{\text {app }}$, that determines the alignment for Er magnetic moments (and thus the magnetization, as the Er moments are an order of magnitude larger than those of Co), and the intra-atomic spin-orbit coupling, $\mathrm{H}_{L S}=\lambda \mathrm{LS}$, that -according to Hund's third rule for more than half-filled shellsfavors a parallel alignment of the Co orbital and spin moments. While $\mathrm{H}_{a p p}$ (in what follows defining the positive direction) was kept constant in the experiment, the second term decreases upon heating from the low temperature phase $\left(T_{\mathrm{c}}<T<T_{\mathrm{F}}\right)$ as both $m_{\mathrm{L}}$ and $m_{\mathrm{S}}$ decrease their values. Both contributions are acting on the Co spin moment in the same way. In addition, two more exchange fields act on the Co spin moment: $\mathrm{H}_{E x}^{C o}$ (created by the Co spins) which favors the parallel alignment of all the Co moments, and $\mathrm{H}_{E x}^{E r}$ (created by Er spin moments) which tends to align the Co moments antiparallel to the Er ones, and consequently to $\mathrm{H}_{a p p}$. This second term is the origin of the ferrimagnetism in $\mathrm{ErCO}_{2}$ at low temperatures. As the inversion of the Co moments takes place in a collective way -via short-range clustering [? ]- $\mathrm{H}_{E x}^{C o}$ does not play a significant role in the field balance, as it always maintains the same sign as the Co spin moments have. On the contrary, $\mathrm{H}_{E x}^{E r}$ on Co is always negative, of the same order of magnitude as $\mathrm{H}_{E x}^{C o}$ [? ], but it decreases upon heating due to the intrinsic thermal disorder of Er magnetic moments in the paramagnetic phase.

The two experimentally discernible temperatures at which the orbital and spin moments of Co go through zero can be explained by taking into account all the fields involved in the process. Upon heating, the relative influence of those fields on $m_{\mathrm{L}}$ and $m_{\mathrm{S}}$ are the following (see also table ??): (a) In the ferrimagnetic phase, $\mathrm{H}_{E x}^{E r}$ is larger than $\mathrm{H}_{a p p}$ and dominant on $m_{\mathrm{S}}$, while $m_{\mathrm{L}}$ is governed by $\mathrm{H}_{L S}$. Both $m_{\mathrm{L}}$ and $m_{\mathrm{S}}$ are oriented antiparallel to $\mathrm{H}_{\text {app }}$, according to Hund's third rule. (b) Above $T_{\mathrm{c}}$ both $\mathrm{H}_{L S}$ and $\mathrm{H}_{E x}^{E r}$ gradually decrease. When $\mathrm{H}_{L S}=\mathrm{H}_{a p p}$, well within the paramagnetic phase, $m_{\mathrm{L}}$ crosses zero $(\mathrm{T} \simeq 59 \mathrm{~K})$. At that temperature $\mathrm{H}_{E x}^{E r}$ is still dominant over $\mathrm{H}_{a p p}+\mathrm{H}_{L S}$, and therefore $m_{\mathrm{S}}$ has the same orientation as in the ferrimagnetic phase, i.e. negative with respect to the applied field. (c) In the temperature region $59 \mathrm{~K} \lesssim \mathrm{T} \lesssim 62 \mathrm{~K}, m_{\mathrm{L}}$ and $m_{\mathrm{S}}$ are oriented antiparallel. On the orbital moment, $\mathrm{H}_{L S}<$ $\mathrm{H}_{a p p}$ but $\mathrm{H}_{E x}^{E r}$ still forces the Co spin moments to be negative. (d) As $\mathrm{H}_{E x}^{E r}$ decreases, the rest of the interactions acting on $m_{\mathrm{S}}$ dominate, and $m_{\mathrm{S}}$ goes through zero at $\mathrm{T} \simeq 62 \mathrm{~K}$ with a subsequent change of sign above that temperature.

Hence, as our experimental results shows, the flipping process from the pari- to the paramagnetic state takes place in two-steps. Upon heating, the Co orbital moment flips to the normal paramagnetic orientation, as it does not feel the internal exchange field due to the Er spins other than via the spin orbit coupling. At a slightly higher temperature, Co spins follow the flipping to paramagnetism. In the temperature range between these two flips, Hund's third rule 
is not obbeyed.

The relationship between the Co orbital and spin moments in $\mathrm{ErCo}_{2}$ can be seen in Fig. ??, where the temperature is implicit and $m_{\mathrm{L}}$ is represented as a function of $m_{\mathrm{S}}$. In the first and third quadrants $m_{\mathrm{L}}$ and $m_{\mathrm{S}}$ are parallel and Hund's third rule is obeyed. The second quadrant corresponds to $m_{\mathrm{L}}$ being positive and $m_{\mathrm{S}}$ negative i.e. the breakdown of Hund's third rule. However, the linear behavior between $m_{\mathrm{L}}$ and $m_{\mathrm{S}}$ across the whole temperature range indicates that this phenomenon is not a local effect due to the reversal of the moment but rather a global effect due to the addition of all interactions present in the system along the complete phase diagram.

Concluding, we have experimentally found a temperature range in which the Co orbital moment is dominated by the external applied field (here stronger than the spin-orbit coupling) while the rather small Co spin moment is governed by the inter-atomic exchange interaction (here stronger than the combined action of the external field and the intra-atomic spin orbit coupling). This scenario gives rise to a "pseudo-violation" of Hund's third rule in the paramagnetic phase of the compound $\mathrm{ErCo}_{2}$. Although Hund's rules should only be strictly obeyed in the atomic framework, it is remarkable that only a few exceptions to their predictions have been found for atoms embedded in solids. Moreover, both the theoretical and experimental exceptions to the Hund's third rule had been only found on induced magnetic moments. To our knowledge this is the first experimental evidence of such phenomenon in an intrinsic magnetic moment. This situation also leads to the observation of other exotic forms of magnetism in solids, such as a "pure" spin or a "pure" orbital moment on the same compound at selected temperature and fields. We hope this experimental result will stimulate further theoretical studies on the balance of intra- and inter-atomic interactions in both intrinsic and induced magnetic moments.

-Financial support from the Spanish MICIN MAT08-1077 and Aragonese CAMRADS projects is acknowledged. Advanced Light Source is supported by the Director, Office of Science, Office of Basic Energy Sciences, of the U.S. Department of Energy under Contract No. DE-AC02-05CH11231-

[] F. Hund, Z. Phys. 33, 345 (1925).

[] F. Hund, Linienspektien p. 124 (1927).

[] J. C. Slater, Phys. Rec. 34, 1293 (1929).

[] A. Hjelm, O. Eriksson, and B. Johansson, Phys. Rev. Lett. 71, 1459 (1993).

[] I. Galanakis and et al., J. Phys. Condens. Matter 13, 4553 (2001).

[] I. Galanakis and et al., Phys. Rev. B 63, 172405 (2001).

[] P. M. Oppeneer and et al., J. Magn. Magn. Mat. 240, 371 (2002).

R. Tyer and et al., Phys. Rev. B 67, 104409 (2003).

[] M. G. Samant and et al., Phys. Rev. 72, 1112 (1994).

[] J. Bartolomé and et al., Phys. Rev. B 77, 184420 (2008).

[] S. Pizzini and et al., Phys. Rev. B 74, 1470 (1995).

[] B. T. Thole and et al., Phys. Rev. Lett. 68, 1943 (1992).

[] P. Carra and et al., Phys. Rev. Lett. 70, 694 (1993).

[] F. Wilhelm and et al., Phys. Rev. Lett. 87, 207202 (2001).

[] X. Qian and W. Hübner, Phys. Rev. B 60, 16192 (1999).

[] X. Qian and W. Hübner, Phys. Rev. B 64, 092402 (2001).

[] I. Galanakis and et al., Phys. Rev. B 62, 3923 (2000).

[] G. Schütz and et al., Phys. Scr. T49A, 302 (1993).

[] V. Krishnamurthy and et al., Phys. Rev. B 74, 064411 (2006).

[] R. Tyer and et al., Phys. Rev. Lett. 90, 129701 (2003).

[] F. Wilhelm and et al., Phys. Rev. Lett. 90, 129702 (2003).

[] H. Wende, Rep. Prog. Phys. 67, 2105 (2004).

[] S. Frota-Pessoa, Phys. Rev. B 69, 104401 (2004).

[] J. Herrero-Albillos and et al., Phys. Rev. B 76, 094409 (2007).

[] J. Herrero-Albillos and et al., Phys. Rev. B 73, 134410 (2006).

[] J. Herrero-Albillos, L. M. García, and F. Bartolomé, J. Phys. Condens. Matter 21, 216004 (2009).

[] A. T. Young, V. Martynov, and H. A. Padmore, J. Electron Spectrosc. Relat. Phenom. 101-103, 885 (1999).

[] A. T. Young and et al, Nucl. Instrum. Methods Phys. Res. A 467-468, 549 (2001).

[] T. Funk and et al., Coord. Chem. Rev. 249, 3 (2005).

[] J. Herrero-Albillos and et al., J. of Applied Physics 103, 07E146 (2008).

[] J. Herrero-Albillos and et al., J. Magn. Magn. Mat. e442-e445, 316 (2007).

[] R. B. Griffiths, Phys. Rev. Lett. 23, 17 (1969).

[] C. T. Chen and et al., Phys. Rev. Lett. 75, 152 (1995).

[] J. Vogel and M. Sacchi, Phys. Rev. B 49, 3230 (1994). 
[] J. Stohr and R. Nakajima, IBM J. Res. Develop. 48, 73 (1998).

F. Luis and et al., Europhysics Letters 76, 142 (2006).

L.M. García and et al., Phys. Rev. Lett. 85, 429 (2000).

F. Bartolomé and et al., J. Magn. Magn. Mat. 272-276, 319 (2004).

[] J. Herrero-Albillos and et al., J. Magn. Magn. Mat. 310, 1645 (2007).

W. Kuch, Physica Scripta T109, 89 (2004).

[] P. Gambardella and et al., Science 300, 1130 (2003). 